\title{
FITTING CLASSES OF CERTAIN METANILPOTENT GROUPS
}

\author{
by HERMANN HEINEKEN
}

\author{
In memory of Hans Zassenhaus
}

(Received 26 October 1992; revised 24 February 1993)

1. Introduction. There are two families of group classes that are of particular interest for clearing up the structure of finite soluble groups: Saturated formations and Fitting classes. In both cases there is a unique conjugacy class of subgroups which are maximal as members of the respective class combined with the property of being suitably mapped by homomorphisms (in the case of saturated formations) or intersecting suitably with normal subgroups (when considering Fitting classes). While it does not seem too difficult, however, to determine the smallest saturated formation containing a given group, the same problem regarding Fitting classes does not seem answered for the dihedral group of order 6 . The object of this paper is to determine the smallest Fitting class containing one of the groups described explicitly later on; all of them are $q p$-groups with cyclic commutator quotient group and only one minimal normal subgroup which in addition coincides with the centre. Unlike the results of McCann [7], which give a determination "up to metanilpotent groups", the description is complete in this case. Another family of Fitting classes generated by a metanilpotent group was considered and described completely by Hawkes (see [5, Theorem 5.5 p. 476]); it was shown later by Brison [1, Proposition 8.7, Corollary 8.8], that these classes are in fact generated by one finite group. The Fitting classes considered here are not contained in the Fitting class of all nilpotent groups but every proper Fitting subclass is. They have the following additional properties: all minimal normal subgroups are contained in the centre (this follows in fact from Gaschütz [4, Theorem 10, p. 64]) and the nilpotent residual is nilpotent of class two (answering the open question on p. 482 of Hawkes [5]), while the quotient group modulo the Fitting subgroup may be nilpotent of any class. In particular no one of these classes consists of supersoluble groups only.

A first step in this direction was done by $M$. Loos [6] who did the determination for $(q, p)=(2,3)$, that is for the minimal non-metabelian group of order 54. This paper includes his result and puts it into a more general setting.

Notation. Unless a particular definition is given, the notation is standard and can be found in any book on finite groups or on soluble groups such as Doerk and Hawkes [3]. All groups considered in this article are finite.

The author is indebted to the referee who suggested many very desirable improvements.

2. Preliminaries. Here we want to collect facts which we will refer to later on and which would disturb the main line of the arguments if proved when needed. Proofs or sketches of proofs are added for the comfort of the reader although these facts should be well known.

LEMMA 1. Let $F$ be a finite field of odd prime order and let $P(a, b)=a^{2}+b^{2}+2 r a b$ be an irreducible polynomial over $F$. Then the set of all values of $P(a, b)$, where $(a, b)$ runs through all ordered pairs of elements in $F$, is $F$ itself. 
Proof. We observe $P(a, b)=(a+r b)^{2}+\left(1-r^{2}\right) b^{2}$. If $1-r^{2}$ is not a square in $F$, then $P(a, 0)$ runs through all squares of $F$ while $P(-r b, b)$ runs through all nonsquares, and so all elements of $F$ occur as values $P(a, b)$. If $1-r^{2}$ is a square, we obtain the result since the squares in $F$ do not constitute an additive subgroup of $F$ and some nonsquare occurs among the $P(a, b)$; and therefore all of them occur.

LEMMA 2. Let $F$ be a finite field. If $M$ is a maximal subgroup of the additive group of $F$ and $c$ is some element of $F$, then $c M=M$ if and only if $c$ belongs to the prime field of $F$.

Proof. Assume that $c M=M$, then likewise $c^{i} M=M$ for all $i$, and if $F^{\prime}$ is the smallest subfield of $F$ that contains $c$, then $M$ may be considered as a vector space over $F^{\prime}$. Now the degree of $F^{\prime}$ over the prime field of $F$ divides the ranks of $M$ and of $F$ and so can only be 1 , showing that $F^{\prime}$ is the prime field in this case.

COROLlaRY 3. The multiplicative group of a finite field $F$ permutes the maximal additive subgroups of $F$ transitively by multiplication.

LemMa 4. Assume that $x$ is an element of prime power order $q$ operating irreducibly and faithfully on an elementary abelian p-group $A$ and that $\operatorname{rank}(A)$ is odd. Choose an isomorphic copy $\left\langle x_{1}, A_{1}\right\rangle$ of $\langle x, A\rangle$ such that the isomorphism maps $x$ onto $x_{1}$. The free nilpotent class two product $F=\left(A * A_{1}\right) /\left(A * A_{1}\right)_{3}$ of $A$ and $A_{1}$ admits an automorphism $\tau$ operating on $A$ like conjugation by $x^{-1}$ and on $A_{1}$ like conjugation by $x_{1}$. The commutator subgroup $F^{\prime}=\left(A * A_{1}\right)^{\prime} /\left(A * A_{1}\right)_{3}$ is a direct product of elementary abelian subgroups which are invariant under $\tau$, and if $W_{1}$ is among the minimal ones on which $\tau$ operates nontrivially, these subgroups $W_{i}$ can be numbered such that $\langle x, A\rangle$ is isomorphic to $\left\langle\tau, W_{i}\right\rangle$ by an isomorphism mapping $x^{1+p^{i}}$ onto $\tau$. The order $F^{\prime} /\left[\tau, F^{\prime}\right]$ is equal to the order of $A$.

LemMa 5. Assume that $x$ is an element of prime power order $q$ operating irreducibly and faithfully on an elementary abelian p-group $Y$ such that $p$ is odd and $\operatorname{rank}(Y)=2 m$ is even. Consider the free (nilpotent class two and exponent $p$ ) group $F$ on $2 m$ generators. Then $F / F^{\prime}$ is isomorphic to $Y$ and there is an automorphism $\tau$ of order $q$ of $F$ that induces an automorphism on $F / F^{\prime}$ such that $\langle x, Y\rangle$ is isomorphic to $\left\langle\tau, F / F^{\prime}\right\rangle$, where the isomorphism maps $x$ onto $\tau$. The commutator subgroup $F^{\prime}$ is a direct product of minimal $\tau$-invariant subgroups $W_{i}$, and if $W_{i}$ is not fixed elementwise by $\tau$, then $\langle x, Y\rangle$ is isomorphic to $\left\langle\tau, W_{i}\right\rangle$, where the isomorphism maps $x^{1+p^{i}}$ onto $\tau$, for suitable $j \neq 0$. Furthermore, the order of $F^{\prime} /\left[F^{\prime}, \tau\right]$ is $p^{m}$.

Lemma 6. Assume that $q$ is a prime and that $m=2 n$ is minimal such that $q$ divides $2^{m}-1$. Let $V$ be the free group on $m$ generators. We consider $F=V /\left(V^{4}\left(V^{\prime}\right)^{2}\left[V, V^{\prime}\right]\right)$ and choose a basis $b_{1}, b_{2}, \ldots, b_{m}$ of $F$. The quotient group $F / F^{2}$ possesses an automorphism $\alpha$ of order $q$. Every mapping $\beta$ of the basis such that $\beta\left(b_{i}\right) F^{2}=\alpha\left(b_{i} F^{2}\right)$ can be extended to an automorphism of $F$ (which we call again $\beta$ ), and its q-th power fixes $F / F^{2}$ and $F^{2}$ elementwise. Now $\beta^{2}$ is an automorphism of $F$, and $F^{2} /\left[F^{2}, \beta^{2}\right]$ has order $2^{n}$.

The reader should note that there is no restriction to the prime $p$ in Lemma 4; the faithful operation yields that $q$ and $p$ are prime to each other. Lemmata 5 and 6 correspond to each other with respect to $p$ being odd or equal to 2 .

Proofs of Lemmata 4 to 6 . The arguments in the nilpotent groups are transformed to the corresponding Lie algebras (first described by Zassenhaus in [9]) and one forms the tensor product with a suitable splitting field. 
3. Some monolithic groups. In this section we formulate the construction of the extension of an extraspecial $p$-group by a cyclic group of order a prime power $t$. We want to achieve the additional properties that every subgroup of $p$-power index is metabelian and that the centre is the only minimal normal subgroup and of order $p$. We distinguish three cases.

Case 1: Let $k$ be minimal such that $t$ divides $p^{k}-1$ and suppose that $k$ is odd.

We denote the field of order $p^{k}$ by $F$. The group $U$ of all upper unitriangular $3 \times 3$ matrices with entries in $F$ is normalized by all $3 \times 3$ diagonal matrices. Choose an element $a$ of $F$ of multiplicative order (exactly) $t$, the diagonal matrix $\mathscr{A}: a_{11}=a_{33}=1, a_{22}=a$ therefore normalizes $U$, it also centralizes $U^{\prime}=Z(U)$. If $\mathscr{B}$ is a matrix belonging to $\langle\mathscr{A}\rangle U$, if further $\mathscr{B}^{*}$ is the inverse of its transpose and $\mathscr{X}$ is the matrix with $x_{13}=x_{22}=-x_{31}=1 ; x_{i j}=0$ otherwise, then the mapping

$$
\varphi: \mathscr{B} \mapsto \mathscr{X}^{-1} \mathscr{B}^{*} \mathscr{X}
$$

is an automorphism of $\langle\mathscr{A}\rangle U$ which inverts $\mathscr{A}$ and fixes every element of $U^{\prime}$. Let $d$ be any non-zero element of $F$ and $\mathscr{C}$ the diagonal matrix with $c_{11}=c_{22}=1, c_{33}=d$. To every pair $R, R^{*}$ of maximal subgroups of $U^{\prime}$ there is an element $d$ such that the matrix $\mathscr{C}$ conjugates one of them into the other (for this see Lemma 2 and the following Corollary). So there is only one isomorphism class of quotient groups $\langle\mathscr{A}\rangle U / R$, and this is the group we are interested in. Notice that the automorphism induced by $\varphi$ fixes the elements of $U^{\prime} / R$ and inverts the $p$-complement.

Case 2: Let $n$ be minimal such that $t$ divides $p^{n}-1$ and suppose that $n$ is even; then $n=2 k$ and $t$ divides $p^{k}+1$.

We have to distinguish here:

Case $2 a: p$ is odd. We consider the field $F$ of order $p^{\prime \prime}$ with element $a$ of multiplicative order $t$. It is well known that $F$ possesses an automorphism of order 2, namely $\sigma: x \rightarrow x^{\left(p^{k}\right)}$. By construction, $a \sigma(a)=1$. We consider the subgroup $V$ of upper unitriangular $3 \times 3$ matrices $\mathscr{H}$ with $h_{12}=\sigma\left(h_{23}\right)$. By the special choice of $a$, this subgroup $V$ is normalized by the diagonal matrix $\mathscr{A}$ constructed from $a$ as in Case 1 , and, as in Case $1, \mathscr{A}$ centralizes $Z(V)=V^{\prime} \times S$, where $S$ is the subgroup of all unitriangular matrices $\mathscr{S}$ with $s_{12}=s_{23}=0, s_{13}=\sigma\left(s_{13}\right)$. Surely $\sigma^{*}$, replacing every position in the matrix by its image under $\sigma$, is an automorphism of $\langle\mathscr{A}\rangle V$ which fixes every element of $S$. Now choose an element $f$ of $F$ which is of order $2\left(p^{k}+1\right)$, so that $f \sigma(f)=-1$, and form the diagonal matrix $\mathscr{B}$ with $b_{11}=f, b_{22}=1, b_{33}=\sigma\left(f^{-1}\right)$. Then the mapping

$$
\varphi: \mathcal{M} \mapsto \mathscr{B}^{-1} \sigma^{*}(\mathcal{M}) \mathscr{B}
$$

induces an automorphism on $\langle\mathscr{A}\rangle V / S$ which inverts $\mathscr{A}$ and fixes $Z(V) / S$ elementwise. If $R / S$ is any maximal subgroup of $Z(V) / S$, then $\langle A\rangle V / R \cong(\langle\mathscr{A}\rangle V / S) /(R / S)$ is the final result of our construction. Again, this is unique up to isomorphism; we see this by conjugating with a suitable diagonal matrix $\mathscr{Y}$ in which $y_{11}=d, y_{22}=1, y_{33}=\sigma\left(d^{-1}\right)$, since the set of elements $d \sigma(d)$ with $d$ in $F$ is the subfield of order $p^{k}$ in $F$, and we again make use of Lemma 2. Furthermore, the quotient group $\langle\mathscr{A}\rangle V / R$ possesses an automorphism fixing the elements of the centre and inverting the $p$-complement: the automorphism induced by $\varphi$ does this. 
Case $2 b: p=2$.

Compared with Case $2 a$, the following alterations are made: We choose an element $g$ such that $g \neq 1=g \sigma(g)$. The matrices $\mathscr{H}$ are chosen unitriangular with $h_{23}=g \sigma\left(h_{12}\right)$, and $\mathscr{B}$ is taken with $b_{13}=b_{22}=b_{31}=1$. The remainder is left unchanged.

Case 3: $t=2^{s}$.

Here we want to consider the case $k=1$ only; in this case the $p$-group in question is the free (class two exponent $p$ ) group with two generators $v, w$, say. It seems easier to consider these generators and their images directly.

Case $3 a: t=2$.

In this case we form the extension of $\langle v, w\rangle$ by an element $x$ with $x^{2}=(x v)^{2}=$ $(x w)^{2}=1$. It follows that $x$ centralizes $([v, w])$.

Case $3 b: t=2^{s}>2$ and $t$ divides $p-1$.

Choose a number $d$ which is of multiplicative order $t$ modulo $p$, and let $c$ be its inverse modulo $p$. Then the extension of $\langle v, w\rangle$ by $x$ such that

$$
x^{\prime}=1 ; \quad x^{-1} v x=v^{d} ; \quad x^{-1} w x=w^{c}
$$

is a construction of the desired form. The prescription

$$
\varphi: x \rightarrow x^{-1}, v \rightarrow w, w \rightarrow v^{-1}
$$

defines an automorphism of $\langle v, w\rangle$ which fixes every element of $\langle[v, w]\rangle$.

Case $3 c: t=2^{s}>2$ and $t$ divides $p+1$.

There is an integer $r$ such that the mapping

$$
\xi: v \mapsto w, \quad w \mapsto v^{-1} w^{2 r}[v, w]^{\alpha}
$$

is of order $t$ for suitable $\alpha$. We form the extension of $\langle v, w\rangle$ by $x$ with $x^{\prime}=1 ; x^{-1} v x=w$; $x^{-1} w x=v^{-1} w^{2 r}[v, w]^{\alpha}$. The polynomial $P(a, b)=a^{2}+b^{2}+2 r a b$ is by construction irreducible modulo $p$. By Lemma 1 there are $c, d$ such that $c^{2}+d^{2}+2 r c d=-1$, and there is an automorphism defined by

$$
\varphi: x \rightarrow x^{-1} ; \quad v \mapsto v^{c} w^{d-2 r c}[v, w]^{\beta} ; \quad w \mapsto v^{d} w^{-c}[v, w]^{\gamma}
$$

with suitable $\beta, \gamma$. This automorphism fixes every element of $\langle[v, w]\rangle$.

The groups we have constructed here will be called $\operatorname{Mon}(t, p)$ from now on, and $\operatorname{Fit}(q, p)$ is the smallest Fitting class containing $\operatorname{Mon}(q, p)$. Comparing the orders of the groups of matrices constructed with those constructed in the previous section, we see that the groups $\operatorname{Mon}(q, p)$ are the unique examples $G$ with the following properties:

(i) $G^{\prime \prime}$ is the unique minimal normal subgroup of $G$, and $G^{\prime \prime}=Z(G)$.

(ii) all proper quotient groups of $G$ are metabelian,

(iii) all proper subgroups $U$ of $G$ satisfying $U G^{\prime}=G$ are metabelian,

(iv) $G / G^{\prime}$ is cyclic of order $q$ and $G^{\prime}$ is a $p$-group.

4. Central products. In the context of this paper a central product is a product of normal subgroups $A_{i}$ such that any of them centralizes the product of the remaining ones. Therefore $A_{i} \cap A_{j} \subseteq Z\left(A_{i}\right)$ for $i \neq j$. In particular, direct products are central products, 
and also extraspecial groups are central products of extraspecial groups of order $p^{3}$. These are the two extremes of the wide range of possibilities.

We will make frequent use of the following fact: If the group $G$ is a central product of normal subgroups $A_{i}$ and $\tau$ is an automorphism of $A_{i}$ which fixes every element of $Z\left(A_{i}\right)$, then we can define an automorphism of $G$ by the following prescription: All elements of $A_{j}$ are left fixed for all $j \neq i$, all elements $y$ of $A_{i}$ are mapped onto $\tau(y)$. The automorphisms constructed in Section 3 in all three cases considered will be seen to be helpful for our reduction argument which we come to now.

Lemma 7. Assume that $G$ is a non-nilpotent extension of a central product of extraspecial $p$-groups by a cyclic group of order a prime $q$ with $q \neq p$; and assume further that the centre of this extension is the product of the centres of all the extraspecial $p$-groups just mentioned. Then the smallest Fitting class containing $G$ contains $\operatorname{Mon}(q, p)$.

Proof. The normal Sylow $p$-subgroup $P$ of $G$ possesses an extraspecial central factor $K$. Denote by $L$ the product of all those central factors of $P$ the commutator subgroups of which coincide with $K^{\prime}$. In this way $P$ can be rearranged as a central product of extraspecial groups with pairwise different commutator subgroups, and each of these factors is $\langle x\rangle$-invariant, where $x$ is an element of order $q$ in $G$. We consider one of these factors $L$; without loss of generality we may assume $[x, L]=L$. Our first step in the proof will be to show that $L$ is the central product of two $\langle x\rangle$-invariant groups $D$ and $C$ such that $\langle x, D\rangle$ is isomorphic to $\operatorname{Mon}(q, p)$. The second step, to show why this group belongs to the same Fitting class, requires different treatment depending on whether $q$ is 2 or odd.

The element $x$ induces by conjugation a linear mapping of order $q$ on the quotient group $L / L^{\prime}$ considered as a vector space over the prime field $F$ of order $p$. Let $L^{\prime}=\langle z\rangle$; the mapping $\left(y_{1} L^{\prime}, y_{2} L^{\prime}\right) \rightarrow k$ whenever $\left[y_{1}, y_{2}\right]=z^{k}$ is known to be an antisymmetric bilinear form of $L / L^{\prime}$, and, by construction, conjugation by $x$ leaves this form invariant.

Let $A / L^{\prime}$ be a minimal $\langle x\rangle$-invariant subgroup of $L / L^{\prime}$. If $A$ is nonabelian, then $P$ is the central product of $A$ and $C_{P}(A)$. Now $P$ is the central product of the two $\langle x\rangle$-invariant subgroups $A=D$ and $C_{P}(A)=C$, also $\langle x, D\rangle$ is isomorphic to $\operatorname{Mon}(q, p)$. If $A$ is abelian and $\operatorname{rank}\left(A / L^{\prime}\right)$ is odd, then there is an $\langle x\rangle$-invariant complement $B / L^{\prime}$ of $C_{L}(A) / L^{\prime}$. Now $P$ is a central product of $A B=D$ and $C_{P}(A B)=C$; and $\langle x, D\rangle$ is isomorphic to $\operatorname{Mon}(q, p)$. If finally $A$ is abelian and $\operatorname{rank}\left(A / L^{\prime}\right)$ is even, we construct a complement $B / L^{\prime}$ as before and consider $A B$. We choose a pair of noncentral elements $a \in A, b \in B$ such that $a$ commutes with $x b x^{-1}$ but not with $x^{-1} b x$ (here $q$ must be odd, so this situation can always be arranged). Now

$$
\begin{aligned}
{\left[a b, x^{-1} a b x\right] } & =\left[a, x^{-1} b x\right]\left[b, x^{-1} a x\right] \\
& =\left[a, x^{-1} b x\right] x^{-1}\left[x b x^{-1}, a\right] x \\
& =\left[a, x^{-1} b x\right] \neq 1 .
\end{aligned}
$$

So $P$ is a central product of $[x,\langle x, a b\rangle]=D$ and $C_{P}(D)=C$, and $\langle x, D\rangle$ is isomorphic to $\operatorname{Mon}(q, p)$.

We will now show that $\langle x, D\rangle$ belongs to the smallest Fitting class containing $G$. First we treat the case $q \neq 2$. We may extend $G$ by an element $y$ such that $y^{q}=1$; $y^{-1} d y=x^{-1} d x$ for all $d \in D ;[x, y]=1$ and $[y, C]=1$. This extension $\langle y, G\rangle$ is a central 
product of $\langle D, y\rangle$ and $\left\langle C, x y^{-1}\right\rangle$, and there is an automorphism $\psi$ of $\langle G, y\rangle$ which fixes $\left\langle C, x y^{-1}\right\rangle$ and maps $\langle D, y\rangle$ as $\varphi$ defined in Section 3 does. In particular,

$$
\psi(G)=\psi(\langle C D, x\rangle)=\left\langle C D, x y^{-2}\right\rangle,
$$

and $\langle G, y\rangle=G \psi(G)$ belongs to the Fitting class of $G$. Now the normal subgroup $\langle D, y\rangle$ of $\langle G, y\rangle$ also belongs to this Fitting class and is isomorphic to $\langle D, x\rangle$.

The second step is more intricate for $q=2$. In this case we may put $D=\langle a, b\rangle$, and we extend $G$ first by elements $u, v$ such that $[u, G]=[v, G]=u^{p}=v^{p}=1 ;[u, v]=[a, b]$. This extension is extended in turn by $y$ and $z$ such that

$$
\begin{gathered}
y^{2}=z^{2}=1 \\
y^{-1} c y=z^{-1} c z=x^{-1} c x \text { for all } c \in C \\
{[y, x]=1 ; z x z=y ; z a z=u ; z b z=v ;} \\
{[y, a]=[y, b]=(y u)^{2}=(y v)^{2}=1 .}
\end{gathered}
$$

Here we assumed $(x a)^{2}=(x b)^{2}=1$; we may without loss of generality. Now the central product $\langle u, v, G\rangle$ is contained in the Fitting class of $G$, and $\langle y, z, u, v, G\rangle$ is generated by the isomorphic subnormal subgroups $\langle u, v, G\rangle=\langle u, v, x, a, b, P\rangle,\langle a, b, y, u, v, P\rangle$ and $\left\langle a u,(v b)^{r}, z, a u^{-1},\left(v^{-1} b\right)^{r}, P\right\rangle$, where $2 r \equiv 1$ modulo $p$. We derive $(x z)^{2}=x y$, and the subnormal subgroup $\langle x z, a, b, u, v\rangle$ of $\langle u, v, G\rangle$ belongs to the smallest Fitting class containing $G$. But $\langle a, b, u, v\rangle$ is a central product of the $\langle x z\rangle$-invariant extraspecial groups $\left\langle a v, b u^{-1}\right\rangle$ and $\left\langle a v^{-1}, b u\right\rangle$. We extend $H=\langle x z, a, b, u, v\rangle$ by $w$ such that $[w, a v]=\left[w, b u^{-1}\right]=[w, x z]=w^{4}=1 ; w^{-1} u w=(x z)^{-1} u(x z)$ for all $u \in\left\langle a v^{-1}, b u\right\rangle$. Now $\langle H, w\rangle$ is a central product of $\left\langle w, a v^{-1}, b u\right\rangle$ and $\left\langle x z w^{-1}, a v, b u^{-1}\right\rangle$. We construct an automorphism $\psi$ of $\langle H, w\rangle$ which fixes the elements of the second factor and maps the elements of the first like the automorphism $\varphi$ defined in Case $3 \mathrm{~b}$ or Case $3 \mathrm{c}$ in Section 3. So $\langle x z, a, b, u, v\rangle=H$ and $\left\langle x z w^{-2}, a, b, u, v\right\rangle$ are isomorphic normal subgroups of $\langle H, w\rangle$ generating $\left\langle H, w^{2}\right\rangle$. Here we find the subnormal subgroup $\left\langle\left(x z w^{-1}\right)^{2}, a v, b u^{-1}\right\rangle$ which is the desired group.

Remark 8. The groups mentioned in Lemma 7 are normal subgroups of central products of $p$-groups and groups $\operatorname{Mon}\langle q, p\rangle$. We see therefore that the smallest Fitting class containing a group mentioned in Lemma 7 coincides with $\operatorname{Fit}(q, p)$.

5. Nilpotent residuals. We recall that the nilpotent residual $G^{*}$ of a group $G$ is the unique normal subgroup $N$ which is minimal with respect to having nilpotent quotient group $G / N$. For normal subgroups $A, B$ of a group $G$ we have $(A B)^{*}=A^{*} B^{*}$. We will now define classes of groups and show that they are Fitting classes.

Lemma 9. Denote by $\Xi$ the class of groups $G$ satisfying the following conditions:

(i) $G$ is a qp-group, where $q$ and $p$ are different primes,

(ii) $G^{*}$ is a central product of extraspecial p-groups,

(iii) $\left[Q, Z\left(G^{*}\right)\right]=1$ for every Sylow $q$-subgroup $Q$ of $G$.

Then $\Xi$ is a Fitiing class. 
Proof. We will show first that normal subgroups of $G$ satisfy the conditions (i)-(iii). Choose a normal subgroup $M$ of $G$, then $M \cap Q=R$ is a Sylow $q$-subgroup of $M$ and $M^{*}=\left[M^{*}, R\right]=\left[G^{*}, R\right]$. By hypothesis, $G^{*}$ is a central product of extraspecial $p$-groups; by combining those factors with coinciding commutator subgroup we can make it a central product of extraspecial groups with pairwise different commutator subgroups. If $T$ is one such factor, then $T Z\left(G^{*}\right)=\left[T Z\left(G^{*}\right), Q\right] Z\left(G^{*}\right)$, and we may replace $T$ by the $Q$-invariant factor $\left[T Z\left(G^{*}\right), Q\right]$. We assume now that this replacement is done for all factors. Now $M^{*}=\left[R, G^{*}\right]$ is the product of the groups $[R, T]$, and so is the product of extraspecial groups, proving the validity of (ii). From $Z\left(M^{*}\right) \subseteq Z\left(G^{*}\right)$ we deduce $\left[R, Z\left(M^{*}\right)\right]=1$, which is (iii), and it is clear that (i) is true.

In our second step we will show that conditions (i)-(iii) are inherited by normal products. Choose two normal subgroups $H$ and $K$ satisfying (i)-(iii). Clearly $H K$ satisfies (i). If $Q$ is a Sylow $q$-subgroup of $H K$, then $Q \cap H=R$ and $Q \cap K=S$ are Sylow $q$-subgroups of $H, K$, respectively. We assume that $H^{*}$ is a central product of $R$-invariant extraspecial factors $T_{i}$ with pairwise different commutator subgroups $\left(T_{i}\right)^{\prime}$. To derive a contradiction we assume the existence of an element $x \in K$ of odd prime power order such that $x^{-1} T_{i} x \neq T_{i}$; we obtain

$$
T_{i} \neq x^{-1} T_{i} x \neq x T_{i} x^{-1} \neq T_{i}
$$

By definition we know that

$$
\left(T_{i}\right)^{\prime} \cap x^{-1}\left(T_{i}\right)^{\prime} x=\left(T_{i}\right)^{\prime} \cap x\left(T_{i}\right)^{\prime} x^{-1}=x^{-1}\left(T_{i}\right)^{\prime} x \cap x\left(T_{i}\right)^{\prime} x^{-1}=1,
$$

and the three conjugates of $T_{i}$ have pairwise trivial intersection and hence centralize each other. Take two elements $y, z$ of $T_{i}$ such that $[y, z] \neq 1$. Now $[x, y]$ and $\left[x^{-1}, z\right]$ are contained in $K^{*}$ since $x$ is contained in $K$ and since $y$ and $z$ are $p$-elements. Now $[[x, y]$, $\left.\left[x^{-1}, z\right]\right] \in\left(K^{*}\right)^{\prime}$ and $\left[\left[[x, y],\left[x^{-1}, z\right]\right], x\right]=1$; on the other hand, $\left[[x, y],\left[x^{-1}, z\right]\right]=[y, z]$, the other three commutators of the expansion are contained in trivial intersections of different conjuates of $T_{i}$. By construction, $[y, x] \in T_{i}$ is different from 1 , and $[[y, z], x] \neq$ 1. This shows that $x$ centralizes $\left(T_{i}\right)^{\prime}$ and, more generally, $Z\left(H^{*}\right)$. If $x$ belongs to $S$ or some conjugate of $S$, we obtain that $Z\left(H^{*}\right)$ is centralized by all conjugates of $S$ and therefore by $S^{K}=S K^{*}$; so $Z\left(H^{*}\right)$ is centralized by $K^{*}$. If $x$ belongs to $K^{*}$, we obtain the same result more directly. By symmetry in $H$ and $K$ we have $Z\left(H^{*}\right) Z\left(K^{*}\right) \subseteq Z\left(H^{*} K^{*}\right)$.

To derive the opposite inclusion, we state first that $K^{*}=\left[K^{*}, R\right] K^{+}$, where $K^{+}=C(R) \cap K^{*}$; this follows from the fact that the orders of $R$ and $K^{*}$ are relatively prime. Now $H^{*} K^{*}=H^{*}\left[K^{*}, R\right] K^{+}=H^{*} K^{+}$since $R \subseteq H$ and so $\left[K^{*}, R\right] \subseteq H^{*}$. Choose any element $f$ of $Z\left(H^{*} K^{*}\right)$; there are elements $f_{1} \in H^{*}$ and $f_{2} \in K^{+}$such that $f=f_{1} f_{2}$. If $h$ is any element of $H^{*}$, we have

$$
1=[f, h]=\left[f_{1} f_{2}, h\right]=f_{2}^{-1}\left[f_{1}, h\right] f_{2}\left[f_{2}, h\right]=\left[f_{1}, h\right]\left[f_{2}, h\right]
$$

and $\left[f_{1}, h^{-1}\right]=\left[f_{2}, h\right]$. Now $\left[f_{1}, h\right] \in\left(H^{*}\right)^{\prime}=Z\left(H^{*}\right)$ and $\left[Z\left(H^{*}\right), R\right]=1$. Therefore

$$
1=\left[\left[f_{1}, h\right]^{-1}, R\right]=\left[\left[f_{2}, h\right], R\right]=h^{-1}\left[f_{2},\left[h^{-1}, R\right]\right] h
$$

and $1=\left[f_{2},\left[H^{*}, R\right]\right]=\left[f_{2}, H^{*}\right]$. Now we have $f=f_{1} f_{2} \in Z\left(H^{*} K^{*}\right)$ and $f_{2} \in C\left(H^{*}\right)$, and $f_{1} \in Z\left(H^{*}\right)$ follows. Consequently $f_{1} \in C\left(K^{*}\right)$ and also $f_{2} \in Z\left(K^{*}\right)$. This shows $Z\left(H^{*}\right) Z\left(K^{*}\right) \supseteq Z\left(H^{*} K^{*}\right)$ and finally

$$
Z\left(H^{*}\right) Z\left(K^{*}\right)=Z\left(H^{*} K^{*}\right)
$$


Certainly $S K^{*}$ is a characteristic subgroup of $K$ and therefore a normal subgroup of $H K$. We obtain $\left[S, Z\left(H^{*} K^{*}\right)\right] \subseteq Z\left(H^{*} K^{*}\right) \cap K^{*}=Z\left(K^{*}\right)$ and therefore $\left[S,\left[S, Z\left(H^{*} K^{*}\right)\right]\right]=1$. Since $S$ and $Z\left(H^{*} K^{*}\right)=Z$ have coprime order, we obtain $[S, Z]=1$, and, by symmetry in $H$ and $K$,

$$
[Q, Z]=1
$$

and we have shown (iii). The Sylow $p$-subgroup $R$ of $H$ is a normal subgroup of $Q$, and $T_{i}=\left[T_{i} Z, R\right]=\left[T_{i} Z, R\right]=\left[T_{i} Z, Q\right]$ by coprime orders of $Q$ and $T_{i} Z$. We see that the factors $T_{i}$ are even $Q$-invariant. By symmetry we have that $K^{*}$ is a central product of $Q$-invariant extraspecial groups $U_{j}$ with pairwise different commutator subgroups. The intersection $T_{i} \cap K^{*}$ is $Q$-invariant but not necessarily $K^{*}$-invariant. Since $H^{*}$ and $K^{*}$ are of orders coprime to that of $Q$, there are $Q$-invariant quotients $A / Z, B / Z, C / Z$ such that

$$
H^{*} / Z=A / Z \times C / Z ; K^{*} / Z=B / Z \times C / Z ; C=\left(H^{*} \cap K^{*}\right) Z \text {. }
$$

It follows that $s^{-1} a s Z=a Z$ for all $a \in A$ and $s \in S, r^{-1} b r Z=b Z$, for all $b \in B$ and $r \in R$; furthermore

$$
[R, A] Z=A ;[S, B] Z=B ;[R, C] Z=[S, C] Z=C .
$$

Since $H^{*} K^{*} / Z$ is a normal product of two abelian groups, it is nilpotent of class two, and all commutators of length four are trivial. It follows that all commutators of length three commute with $Q H^{*} K^{*}$. We will use this to show that all commutators of length three are trivial. For this it suffices to have the entries of the commutator belonging to $A, B$, or $C$; we will call the entries then $a_{i}, b_{j}, c_{k}$, respectively.

Since $[c, b] \in Z$, we have

$$
[[c, b], a]=\left[\left[c, b_{1}\right], b_{2}\right]=\left[\left[c_{1}, b\right], c_{2}\right]=1 .
$$

By symmetry,

and so

$$
\left[[c, a], H^{*} K^{*}\right]=1,
$$

$$
\begin{gathered}
{[[a, b], c]=[[c, b], a][[c, a], b]^{-1}=1,} \\
{\left[\left[a_{1}, a_{2}\right], H^{*} K^{*}\right]=\left[\left[b_{1}, b_{2}\right], H^{*} K^{*}\right]=\left[\left[c_{1}, c_{2}\right], H^{*} K^{*}=1 .\right.}
\end{gathered}
$$

For any element $s \in S$ we obtain

$$
\left[\left[b, a_{1}\right], a_{2}\right]=s^{-1}\left[\left[b, a_{1}\right], a_{2}\right] s=\left[\left[s^{-1} b s, a_{1}\right], a_{2}\right]
$$

and so $\left[\left[[s, b], a_{1}\right], a_{2}\right]=1 ;$ so $1=[[[S, B], A], A]=[[B, A], A]$ and again $\left[\left[b, a_{1}\right], a_{2}\right]=1$.

By analogy, substituting $(B, A, R)$ for the triple $(A, B, S)$, we find $\left[\left[a, b_{1}\right], b_{2}\right]=1$. This completes the argument to show that $H^{*} K^{*}$ is nilpotent of class two. Having achieved this, we find $[a, b]=r^{-1}[a, b] r$ and $\{[R, A], B]=[A, B]=1$; also $\{[R, C], B]=$ $[C, B]=1$ and $[[S, C], A]=[C, A]=1$. Now $H^{*} K^{*}$ is a central product of $A, B$ and $C$ which in turn are central products of ( $Z$ and) the intersections $T_{i} \cap A, T_{i} \cap C, U_{j} \cap C$, $U_{j} \cap B$. Each of these are extraspecial groups. This shows (ii).

6. Central wreath products. If $A$ and $B$ are two finite groups, we denote their (standard) wreath product by $A \backslash B$. This is a split extension of the direct product of $|B|$ many copies of $A$ by $B$. The direct product is replaced by a central product with identified centres if we consider the quotient group $\left(A\lceil B) /\left[Z(A)^{B}, B\right]\right.$ instead. This is what we call 
a central wreath product and we will denote it by $A \prod_{c} B$ for short. In particular, if $A$ is extraspecial, $A \chi_{c} B$ is the split extension of an extraspecial group by $B$.

Assume that $G$ is the extension of an extraspecial $p$-group $P$ by a $q$-group $Q$ such that $[Z(P), Q]=1$. Then the group of automorphisms induced by conjugation in $P$ is isomorphic to a subgroup of some Sylow $q$-subgroup of the symplectic group $\operatorname{Sp}(2 n, p)$, where $2 n$ is the rank of $P$. The Sylow $q$-subgroups themselves are known for odd $q$ to be isomorphic to the direct product of suitable iterated wreath products $\left(\ldots\left(\left(C_{q^{\prime}} \backslash C_{q}\right) \backslash C_{q}\right) \ldots C_{q}\right)$ where $q^{r}$ is the highest power of $q$ that divides a number $p^{s}-1$ with $s$ not divisible by $q$. If for instance $s$ as defined above is minimal and even, then the Sylow $q$-subgroup of $\operatorname{Sp}\left(s q^{m}, p\right)$ is isomorphic to the wreath product with $m$ iterations. This can be found in Weir [7]. The corresponding split extension of the extraspecial $p$-group of rank $s q^{m}$ by this Sylow $q$-subgroup with the corresponding operation by conjugation is then isomorphic to $\left(\ldots\left(\left(\operatorname{Mon}\left(q^{r}, p\right) l_{c} C_{q}\right) l_{c} C_{q}\right) \ldots C_{q}\right)$ with also $m$ iterations. For other ranks the group is a central product with identified centres with factors of this form.

We want to show that extensions of extraspecial $p$-groups $P$ by $q$-groups $Q$ with $[Z(P), Q]=1$ belong to the Fitting class Fit $(q, p)$. We begin with a special case.

LEMMA 10. If $q$ and $p$ are different primes, $q$ is odd and $q^{r}$ is the highest power of $q$ which divides $p^{s}-1$ where $s$ is minimal such that $q \mid\left(p^{s}-1\right)$, then $\left(\ldots\left(\left(\operatorname{Mon}\left(q^{r}, p\right) l_{r} C_{q}\right) l_{c} C_{q}\right) \ldots C_{q}\right)$ is contained in $\operatorname{Fit}(q, p)$ for any number of iterations.

Proof. By definition, $\operatorname{Mon}(q, p)$ belongs to $\operatorname{Fit}(q, p)$. For our iteration we need the following rule:

$(+)$ If $H$ is the extension of an extraspecial $p$-group by a $q$-group and $H$ belongs to $\operatorname{Fit}(q, p)$, then so does $H l_{c} C_{q}$.

Assume that $R$ is the normal Sylow $p$-subgroup of $H$. Then $R l_{c} C_{q}$ belongs to Fit $(q, p)$ by Lemma 7 and is subnormal in $H l_{c} C_{q}$. Also the central product of the conjugates of $H$ belongs to Fit $(q, p)$, and so does their product. This proves statement $(+)$.

If now $m$ is an integer smaller than $r$ and such that $\operatorname{Mon}\left(q^{m}, p\right)$ belongs to $\operatorname{Fit}(q, p)$, then so does $\operatorname{Mon}\left(q^{m}, p\right){ }_{c} C_{q}$. We consider the subgroup $W$ generated by the full $p$-subgroup $P$ and some cyclic subgroup of order $q^{m+1}$. This is a subnormal subgroup, and again belongs to $\operatorname{Fit}(q, p)$. Since $m$ is smaller than $r$, the normal Sylow $p$-subgroup splits into a central product of $W$-invariant extraspecial $p$-subgroups; let $V$ be a minimal one among them so that $P$ is the central product of $V$ and $U$, and let $x$ be some element of order $q$. We extend $W$ by some element $y$ of order $q$ centralizing $U$ and satisfying $y^{-1} v y=x^{-1} v x$ for all $v \in V$. Let $x y^{-1}=z$; then $\langle W, y\rangle$ is the central product $\langle V, y\rangle\langle U, z\rangle$. We have shown in Section 3 that there is an automorphism of $\langle V, y\rangle \cong \operatorname{Mon}(q m+1, p)$ which inverts $y$ and leaves $V^{\prime}$ fixed elementwise. So $W=$ $\langle V, U, y z\rangle \cong\left\langle V, U, y^{-1} z\right\rangle$, and $\langle W, y\rangle$ belongs to $\operatorname{Fit}(q, p)$ since it is a normal product of $W$ and $\left\langle V, U, y^{-1}\right\rangle$, and also $\langle V, y\rangle$ belongs to $\operatorname{Fit}(q, p)$. We have shown

$(++)$ If $\operatorname{Mon}\left(q^{m}, p\right) \in \operatorname{Fit}(q, p)$ and $m$ is smaller than $r$, then also $\operatorname{Mon}\left(q^{m+1}, p\right) \in$ $\operatorname{Fit}(q, p)$. 
It is now obvious that the statement of the Lemma follows from $(+)$ and $(++)$.

We will now treat the case $q=2$ accordingly. Here the Sylow 2-subgroup of the symplectic group $\operatorname{Sp}\left(2^{n}, p\right)$ is isomorphic to the wreath product $\left(\ldots\left(\left(D \backslash C_{2}\right) \backslash C_{2}\right) \ldots \backslash C_{2}\right)$, where the number of iterations is $n-1$ and $D$ is the (generalized) quaternion group of order $2^{r}$, where $r$ is maximal such that $2^{r}$ divides $p^{2}-1$ (see Carter and Fong [2], p. 142-3). The monolithic group which is the extension of a nonabelian group of order $p^{3}$ by $D$ we will denote by $\operatorname{Mon}^{\dagger}\left(2^{r}, p\right)$ with a dagger $\dagger$ as a reminder that the Sylow 2-subgroup is not cyclic. Now we can formulate the corresponding statement.

LEMMA 11. If $2^{r}$ is the highest power of 2 dividing $p^{2}-1$, then

$$
\left(\ldots\left(\left(\operatorname{Mon}^{\dagger}\left(2^{r}, p\right) l_{c} C_{2}\right) l_{r} C_{2}\right) \ldots l_{r} C_{2}\right) \in \operatorname{Fit}(2, p)
$$

for any number of iterations.

Proof. As a first step we obtain as in Lemma 10.

( + ) If $H \in \operatorname{Fit}(2, p)$ is the extension of an extraspecial $p$-group by a 2-group, then also $H \prod_{c} C_{2} \in \operatorname{Fit}(2, p)$.

The proof is the same as in Lemma 10 and is therefore omitted. As a second statement we will need

$(++) \operatorname{Mon}(4, p) \in \operatorname{Fit}(2, p)$.

To show this we use $(+)$ to see that $\operatorname{Mon}(2, p) l_{c} C_{2} \in \operatorname{Fit}(2, p)$. There is a subgroup of index 2 in this central wreath product which is an extension of an extraspecial $p$-group of order $p^{5}$ by a cyclic group of order 4 , like $L=\langle x, a, b, c, d\rangle$ satisfying the conditions

$$
\begin{gathered}
x^{4}=a^{p}=b^{p}=c^{p}=d^{p}=[a, b][d, c]=1, \\
x^{-1} a x=b, x^{-1} b x=a^{-1}, x^{-1} c x=d, \\
x^{-1} d x=c^{-1},[u,[a, b]]=1 \text { for } u=x, a, b, c, d .
\end{gathered}
$$

There are integers $v, w$ such that $v^{2}+w^{2} \equiv-1$ modulo $p$. The reader will find that the extension $\langle z, L\rangle$ with

$$
\begin{gathered}
z^{-1} a z=a^{v} b^{w}, z^{-1} b z=a^{w} b^{-v} \\
z^{-1} c z=d^{-1}, z^{-1} d z=c, x^{2}=z^{2}
\end{gathered}
$$

is generated by the isomorphic normal subgroups $\langle x, a, b, c, d\rangle$ and $\langle z, a, b, c, d\rangle$ which has the normal subgroup $\langle x z, a, b\rangle \cong \operatorname{Mon}(4, p)$, showing statement $(++)$. Since the generalized quaternion group is generated by its (cyclic) subgroups of order 4 , we have that $\operatorname{Mod}^{\dagger}\left(2^{r}, p\right)$ is generated by subnormal subgroups isomorphic to $\operatorname{Mon}(4, p)$. This shows

$$
(+++) \operatorname{Mon}^{\dagger}\left(2^{r}, p\right) \in \operatorname{Fit}(2, p) \text {. }
$$

The proof now follows from $(+)$ and $(+++)$.

Corollary 12. Assume that $K$ is an extension of an extraspecial p-group $P$ by a $q$-group $Q$ where $p$ and $q$ are different primes and $[Q, Z(P)]=1$. Then $K \in \operatorname{Fit}(q, p)$. 
For a proof denote the normal Sylow $p$-subgroup of $K$ by $P$, and fix some Sylow $q$-subgroup $Q$. Then $K /(Q \cap C(P))$ is isomorphic to a central product of groups mentioned in Lemma 10 and 11 respectively, so $K /(Q \cap C(P)) \in \operatorname{Fit}(q, p)$. Furthermore, $Q \in \operatorname{Fit}(q, p)$, and the same is true for $K$ which is isomorphic to some subnormal subgroup of $(K /(Q \cap C(P))) \times Q$.

\section{The final result.}

TheOREM 13. Fit $(q, p)$ is the class of groups satisfying the conditions (i)-(iii) of $\Xi$ in Lemma 9.

Proof. The group $H \in \Xi$ is a normal product of its Sylow $p$-subgroup $P$ and the product $H^{*} Q$, where $Q$ is some Sylow $q$-subgroup. Clearly $P \in \operatorname{Fit}(q, p)$. By (ii), $H^{*}$ is the central product of $Q$-invariant extraspecial $p$-groups $T_{i}$, and therefore $H^{*} Q$ is isomorphic to a subnormal subgroup of a central product of factors $Q T_{i}$ arranged in such a way that its restriction to the $T_{i}$ leads to $H^{*}$. This shows that also $H^{*} Q \in \operatorname{Fit}(q, p)$ is true, and so finally $H \in \operatorname{Fit}(q, p)$.

Remark 14. Theorem 13 and Lemma 9 taken together show that the classes $\Xi$ have all their proper subclasses included in the class of nilpotent groups. In particular, we have a description of the Fitting class generated by $\operatorname{Mon}(2, p)$ for odd $p$, which are groups of order $2 p^{3}$, and also the Fitting class generated by $\operatorname{Mon}(3,2) \cong \operatorname{SL}(2,3)$ is defined.

\section{REFERENCES}

1. O. J. Brison, Relevant groups for Fitting classes, J. Algebra 68 (1981), 31-53.

2. R. W. Carter and P. Fong, The Sylow 2-subgroups of the finite classical groups, J. Algebra 1 (1964), 139-151.

3. K. Doerk and T. O. Hawkes, Finite Soluble Groups. (De Gruyter, Berlin, New York 1992).

4. W. Gaschütz, Lectures on subgroups of Sylow type in finite soluble groups. Notes on Pure Mathematics 11 (ANU, Canberra 1979).

5. T. O. Hawkes, On metanilpotent Fitting classes, J. Algebra 63 (1980), 459-483.

6. M. Loos, Die von einer Gruppe der Ordnung 54 erzeugte Fittingklasse. Diploma Thesis, Würzburg 1992.

7. B. McCann, Examples of minimal Fitting classes of finite groups. Arch. Math. 49 (1987), $179-186$.

8. A. J. Weir, Sylow $p$-subgroups of the classical groups over finite fields with characteristic prime to $p$, Proc. Amer. Math. Soc. 6 (1955), 529-533.

9. H. Zassenhaus, Ein Verfahren, jeder endlichen p-Gruppe einen Lie-Ring mit der Charakteristik p zuzuordnen, Abh. Math. Sem. Univ. Hamburg 13 (1939), 200-207.

Mathematisches Institut

UNIVERSITÄT WÜRZBURG

AM HUBLAND

8700 WÜRZBURG

GERMANY 